\title{
Investigation of early and lifetime clinical features and comorbidities for the risk of developing treatment-resistant depression in a 13-year nationwide cohort study
}

Shiau-Shian Huang ${ }^{1,2,3,4}$, Hsi-Han Chen ${ }^{5}$, Jui Wang ${ }^{1}$, Wei J. Chen ${ }^{1,6,7}$, Hsi-Chung Chen ${ }^{6 *}$ and Po-Hsiu Kuo ${ }^{1,6^{*}}$ (D)

\begin{abstract}
Background: To investigate the risk of treatment-resistant depression (TRD) in patients with depression by examining their clinical features, early prescription patterns, and early and lifetime comorbidities.
\end{abstract}

Methods: In total, 31,422 depressive inpatients were followed-up from diagnostic onset for more than 10-years. Patients were diagnosed with TRD if their antidepressant treatment regimen was altered $\geq$ two times or if they were admitted after at least two different antidepressant treatments. Multiple Cox regression model were used to determine whether physical and psychiatric comorbidities, psychosis, and prescription patterns increased the risk of TRD by controlling for relevant demographic covariates. Survival analyses were performed for important TRD-associated clinical variables.

Results: Females with depression (21.24\%) were more likely to suffer from TRD than males (14.02\%). Early anxiety disorders were more commonly observed in the TRD group than in the non-TRD group (81.48 vs. $58.96 \%, p<0.0001)$. Lifetime anxiety disorders had the highest population attributable fraction (42.87\%). Seventy percent of patients with multiple psychiatric comorbidities developed TRD during follow-up. Cox regression analysis further identified that functional gastrointestinal disorders significantly increased TRD risk ( $a H R=1.19)$. Higher doses of antidepressants and benzodiazepines and $Z$ drugs in the early course of major depressive disorder increased TRD risk $(p<0.0001)$.

Conclusion: Our findings indicate the need to monitor early comorbidities and polypharmacy patterns in patients with depression associated with elevated TRD risk.

Keywords: Treatment-resistant depression, Antidepressants, Early and lifetime comorbidities, Prescription pattern, Healthseeking behavior

\section{Background}

Major depressive disorder (MDD) is a common mental disorder with high lifetime prevalence [1]. The average age of onset for MDD ranges from the patient's early twenties to their late thirties [1]. The symptoms of MDD

\footnotetext{
* Correspondence: hsichungchen@ntu.edu.tw; phkuo@ntu.edu.tw

${ }^{6}$ Department of Psychiatry, National Taiwan University Hospital, Taipei, Taiwan

${ }^{1}$ Department of Public Health \& Institute of Epidemiology and Preventive Medicine, National Taiwan University, Taipei, Taiwan

Full list of author information is available at the end of the article
}

significantly impair patients' daily functions and a substantial proportion of patients with MDD do not respond to standard antidepressant treatment regimens [1]. Patients who do not respond to antidepressants are likely to suffer from treatment-resistant depression (TRD) [2]. Treatment resistance increases medical burden and individual and societal costs [3] The systematic evaluation of TRD and understanding its risk profiles, disease mechanism, prescription patterns, and the impacts on the mental health

C C The Author(s). 2020 Open Access This article is licensed under a Creative Commons Attribution 4.0 International License, which permits use, sharing, adaptation, distribution and reproduction in any medium or format, as long as you give appropriate credit to the original author(s) and the source, provide a link to the Creative Commons licence, and indicate if changes were made. The images or other third party material in this article are included in the article's Creative Commons licence, unless indicated otherwise in a credit line to the material. If material is not included in the article's Creative Commons licence and your intended use is not permitted by statutory regulation or exceeds the permitted use, you will need to obtain permission directly from the copyright holder. To view a copy of this licence, visit http://creativecommons.org/licenses/by/4.0/. The Creative Commons Public Domain Dedication waiver (http://creativecommons.org/publicdomain/zero/1.0/) applies to the data made available in this article, unless otherwise stated in a credit line to the data. 
system may help in developing strategies to prevent the adverse consequences associated with TRD.

The criteria for TRD has been defined by European Medicines Agency as the failure to benefit from at least two different antidepressant treatments with sufficient duration at an adequate dose [4-7]. The proportion of patients with TRD among those with MDD varies between studies (ranging from 6 to 50\% according to databased analyses vs. clinical studies) due to differences in study designs and definitions of TRD [8]. Patients with TRD tend to have more frequent hospitalization, recurrent depressive episodes, and emergency room (ER) visits $[2,6,9]$. In addition, outpatient care is insufficient for a substantial proportion of patients with MDD despite advances in clinical care, treatment regimens, and drug development. Approximately $8.3 \%$ of patients with MDD are hospitalized annually [10]. These patients often exhibit worse symptoms, increased comorbidities, and higher suicide risk, and are prescribed higher doses of antidepressant [11]. MDD patients who were ever admitted to the psychiatric ward represent a prominent subgroup requiring intense care and complex treatment regimens; they are at a high risk of developing TRD.

Several clinical and psychosocial factors have been reported to be associated with poor treatment response in patients with MDD, including poor treatment adherence, physical and psychiatric comorbidities, bipolarity, and high level of neuroticism [6, 12-14]. Physical comorbidities, including diabetes $[15,16]$, heart diseases [17], functional gastrointestinal disorders (FGIDs) [18], thyroid disease [19], systemic lupus erythematosus (SLE) [20], and rheumatoid arthritis [21], have been implicated in the development and prognosis of common mental disorders, especially mood disorders. In addition, a group of anxiety disorders (including panic disorder, generalized anxiety disorder, social anxiety disorder, and obsessive-compulsive disorder), attention deficit hyperactivity disorder (ADHD), substance use disorders (SUDs), and personality disorders were found to be more prevalent in patients with MDD [22-25], or predictive of worse outcomes or slower remission [24]. This emergent evidence has indicated that both physical and psychiatric comorbidities might prolong the course of depression and worsen clinical outcomes. However, the magnitude of impacts of comorbidities in disease onset or during disease course on treatment resistance in depression are not well studied for MDD. For the former, a prospective study design is required to evaluate effects of early comorbidity status on the risk of developing TRD.

Patients taking antidepressants often have poor adherence, resulting in inadequate treatment effects and a high risk of developing TRD $[8,14,26]$. In clinical settings, it would be helpful to recognize the patterns of healthseeking behavior and medical prescriptions during the onset or early in the course of MDD to improve the management of TRD patients. We used a large-scale claims-based database covering the majority of psychiatric patients ever admitted to a hospital to minimize selection bias and compared the results with those of ordinary observational studies or clinical trials. In this database, MDD patients were followed-up from the onset of diagnosis until year 2011. We quantified the proportion of TRD in the nationwide database, evaluated the general characteristics of TRD, and investigated the risk profiles. We examined the effects of physical and psychiatric comorbidities in lifetime, occurring at the onset, or during the course of MDD on the risk of developing TRD. There were several goals in the present study. First, we used a retrospective study design to evaluate feature of TRD in lifetime. Second, we aimed to evaluate the influence of early prescription patterns, health-seeking behavior, and the severity of depressive symptoms during the first year of MDD diagnosis in patients who developed TRD versus those who did not develop TRD during follow-up. Third, using a prospective study design and analytical approaches, the influence of comorbidities on the risk of TRD was investigated using the time-to-event (TRD occurring) method during 13-years of follow-up in patients with MDD. Lastly, the population attributable fraction (PAF) was calculated to estimate the impact of important comorbidities associated with TRD.

\section{Methods}

\section{Data source and study population}

The national health insurance program was launched in 1995 to finance healthcare for all individuals in Taiwan and covered approximately $97 \%$ of the population. The national health insurance research database contains the Psychiatric Inpatients Medical Claim (PIMC) dataset, which includes all patients who were ever hospitalized for any psychiatric diagnosis $(n=187,117)$. The PIMC dataset includes basic demographic information and comprehensive medical records (e.g., medication, outpatient service, and emergency visit) [27]. Individuals included in the PIMC dataset with a principal inpatient diagnosis of MDD (ICD-9-CM code 296.2-3, $n=39,353$ ) between 1996 and 2011 were eligible for inclusion in the present study (Fig. 1). We targeted patients who had ever been admitted to a hospital because of depressive episodes. Patients were excluded if (1) MDD diagnosis was not made by a psychiatrist, (2) if they were diagnosed with schizophrenia (ICD-9-CM code 295) or bipolar disorder (BpD) (ICD-9CM code 296.0, 296.1, and 296.4-8) before MDD diagnosis, or (3) patients have not been admitted for diagnosis of MDD after 1999. All medical records, and diagnoses of physical and psychiatric comorbidities based on ICD-9CM codes were then obtained for MDD patients during the whole study period. Taken together, 31,422 patients with MDD were included in the present study. The longest follow-up duration was 13 years. 


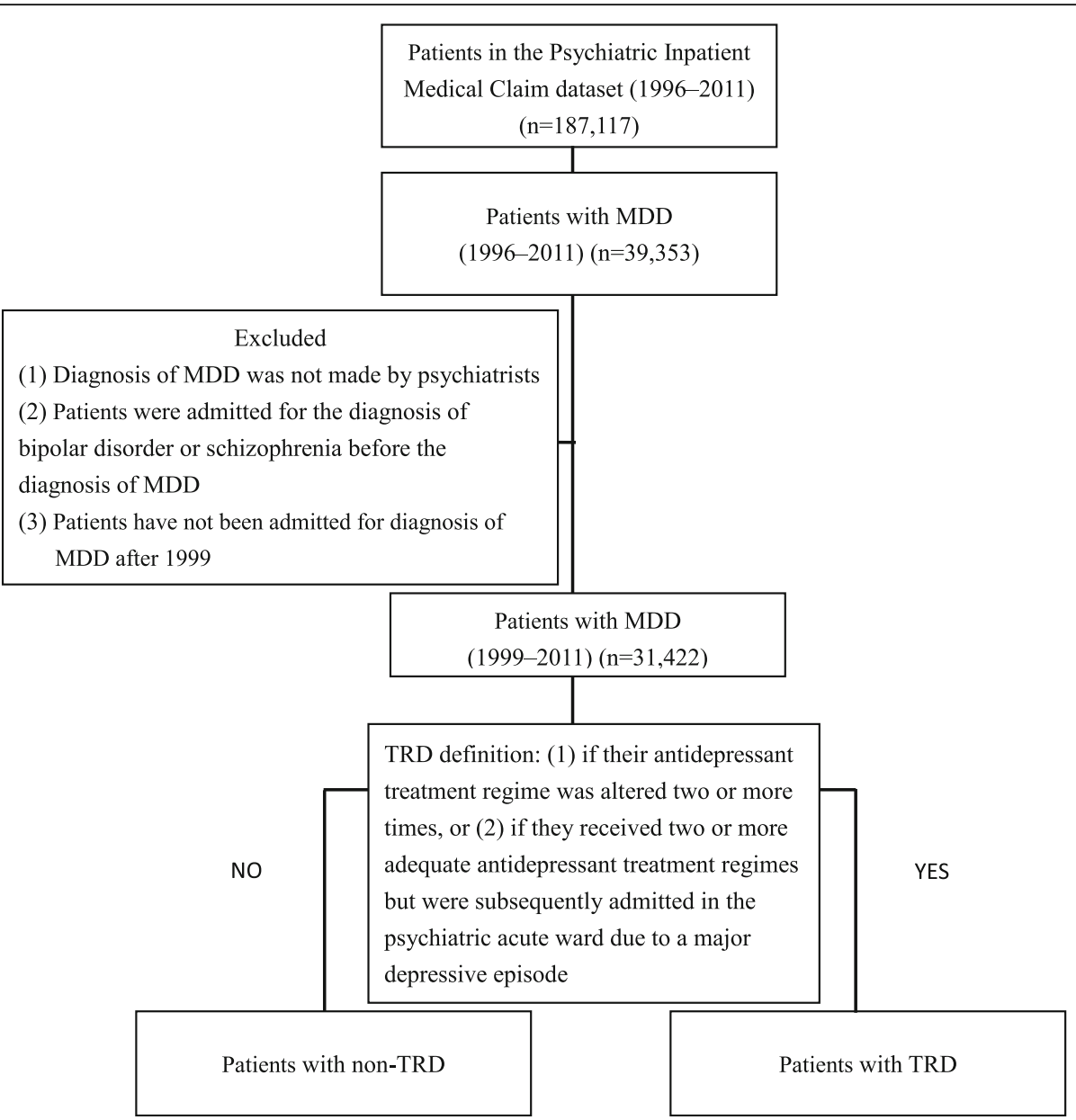

Fig. 1 Consort diagram of participants' selection. Abbreviations: TRD, treatment-resistant depression; MDD, major depressive disorder

\section{Operational definition of TRD}

Depression is usually classified as TRD when at least two treatments of different antidepressants (adequate in terms of dosage and duration) fail to produce a significant clinical improvement. In the present study, we defined patients as treatment-resistant (1) if their antidepressant treatment regimen was altered two or more times for different antidepressants, or (2) if they received two or more different adequate antidepressant treatment regimens but were subsequently admitted to the psychiatric acute ward due to a major depressive episode $[4,7]$. An adequate antidepressant trial was defined as having greater than 56 cumulative defined daily doses (DDD) for each antidepressant treatment over at least 8 consecutive weeks $[6,28]$.

\section{Independent variables and covariates}

Demographic and clinical data between TRD and nonTRD groups were extracted from PIMC. The demographic features were age, sex, urbanization level, and income (estimated via insurance). The urbanization level of patients was stratified as seven levels, with level I being the most urbanized and level VII being the least urbanized [29]. Clinical information included psychiatric and physical comorbidities, prescription patterns, records of medical utility, all-cause mortality, conversion rate to another diagnosis during follow-up, and time from the MDD diagnosis to another diagnosis. Data of physical comorbidities were collected: diabetes, SLE, rheumatoid arthritis, cardiovascular disease, renal disease, FGIDs, and thyroid dysfunction. The psychiatric comorbidities were personality disorders, ADHD, SUDs, anxiety disorders, panic disorders, and nonorganic psychosis. In terms of medical utility, we collected information on the number of ER visits, admissions to all specialized departments, and outpatient visits for patients diagnosed with MDD. During the long-term follow-up, a patient with an original diagnosis of MDD may be diagnosed with other disorders, such as BpD and schizophrenia [12], which might contribute to difficulties in original treatment using antidepressants; therefore, the conversion rate to $\mathrm{BpD}$ or schizophrenia was calculated. The all-cause mortality rate was also evaluated to indicate the long-term prognosis of patients with or without TRD development. 
All comorbidities in the claims database were defined by at least three outpatient visits or one inpatient care visit that was linked to the diagnosis. Early psychiatric and physical comorbidities were defined as follows, those occurring before MDD diagnosis or within 1 year after MDD diagnosis. Furthermore, the severity of depression at the first year of MDD diagnosis was recorded. Severe depression was determined based on the following criteria: 1) patient diagnosis matched ICD-9-CM codes: $296.23,296.24,296.33$ or 296.34 , or 2 ) the patient received an electroconvulsive therapy intervention during a depressive episode [30]. Prescription patterns during the first year following MDD onset and the information on medical care-seeking behavior were also evaluated. The average doses of antidepressants, lithium/mood stabilizers, and antipsychotics, as well as benzodiazepines and $\mathrm{Z}$ Drugs were determined. Early prescription pattern of combined treatment regimens (antidepressants and either antipsychotics or anticonvulsants) within 1 year of onset was analyzed.

\section{Statistical analysis}

All analyses were performed using SAS, version 9.3. Differences between TRD and non-TRD groups were evaluated using the chi-squared test for categorical variables and the t-test for continuous variables. Bonferroni correction was used for multiple comparisons. Multiple Cox regression models were used to examine the effects of demographic variables and clinical features on the risk of developing TRD. The variables examined were sex, physical comorbidities, psychiatric comorbidities, diagnosis conversion, dose of antidepressants and benzodiazepines within 1 year since MDD onset, and the number of non-psychiatric outpatient visits 1 year before or after the onset of depression while adjusting for demographic covariates in the model (age, geographic area, urbanization level, and income estimated via insurance). Adjusted hazard ratios (aHRs) and 95\% P for each covariate were reported for regression analyses. PAF for each significant comorbidity was assessed for the presence of TRD using Levin's formula [31].

Survival analyses (from the onset of MDD to the occurrence of TRD) were performed to evaluate the distribution and proportion of TRD cases stratified by sex, comorbid FGIDs, comorbid thyroid dysfunction, severity of depressive symptoms within 1 year of onset, non-organic psychosis, and psychiatric comorbidities (anxiety disorders, SUDs, and personality disorders). Individuals who did not develop TRD, died, or dropped out were censored. A two-sided $p$ value of $<0.05$ was considered statistically significant.

To evaluate the robustness of the study, sensitivity analysis was performed. In this analysis, early comorbidities were defined as only those occurring before the MDD onset. There were no significant differences between the different definitions.

\section{Results}

The demographic and clinical characteristics of the patients are presented in Table 1. A total of 11,078 of 31,422 (35.26\%) inpatients with MDD included in the study were defined as having TRD (21.24\% of female and $14.02 \%$ of male patients). Females accounted for the majority of TRD patients than non-TRD patients $(60.24 \%$ vs. $39.56 \%, p<$ 0.0001). The age of MDD onset was higher in the TRD group than in the non-TRD group $(41.31 \pm 15.33$ vs. $37.85 \pm 18.82, p<0.0001)$. A greater percentage of patients in the TRD group had no income compared with that of those in the non-TRD group ( $21.82 \%$ vs. $16.3 \%, p<0.0001)$. Patients with TRD exhibited a higher frequency of physical comorbidities, including diabetes, SLE, rheumatoid arthritis, cardiovascular disease, renal disease, FGIDs, and thyroid dysfunction, than non-TRD patients. All psychiatric comorbidities except ADHD were more prevalent in the TRD group than in the non-TRD group; these psychiatric comorbidities were personality disorders $(24.34 \%$ vs. $15.07 \%$, $p<0.0001)$, SUDs $(33.03 \%$ vs. $16.64 \%, p<0.0001)$, anxiety disorders $(89.84 \%$ vs. $64.70 \%, p<0.0001)$, panic disorder ( $15.63 \%$ vs. $6.02 \%, p<0.0001$ ), and non-organic psychosis ( $48.02 \%$ vs. $32.67 \%, p<0.0001)$. During follow-up, a high proportion of patients with TRD had their diagnosis converted to $\mathrm{BpD}(22.53 \%$ vs. $10.57 \%, p<0.0001)$ or schizophrenia $(9.51 \%$ vs. $7.38 \%, p<0.0001)$. Patients with TRD utilized medical care more frequently across all specialties than non-TRD patients. Overall medical care utilization was determined by calculating the number of hospital admissions plus ER visits $(6.85 \pm 15.17$ vs. $2.40 \pm 4.09, p<$ 0.0001 ) (Table 1). The average time from the onset of depression to TRD diagnosis was 3.32 years.

The early characteristics of patients with TRD are presented in Table 2. Generally, patients with TRD exhibited greater early physical comorbidities than patients without TRD. For early psychiatric comorbidities, patients with TRD had a higher prevalence of SUDs (15.68\% vs. $10.58 \%$, $p<0.0001)$, anxiety disorders $(81.48 \%$ vs. $58.96 \%, p<$ $0.0001)$, and panic disorder $(6.51 \%$ vs. $3.57 \%, p<0.0001)$ than patients without TRD. However, patients with TRD had a lower prevalence of early personality disorders ( $8.34 \%$ vs. $10.64 \%, p<0.0001$ ), early non-organic psychosis (7.23\% vs. $11.98 \%, p<0.0001)$, and symptoms of severe depression within 1 year of onset $(23.8 \%$ vs. $33.97 \%, p<$ $0.0001)$. A higher percentage of patients in the TRD group had their diagnosis converted to $\mathrm{BpD}(13.15 \%$ vs. $10.26 \%$, $p<0.0001)$. Among all inpatients with MDD, $22.8 \%$ did not receive adequate treatment with antidepressants, antipsychotics, or anticonvulsants for 1 year since the onset of depression. During the first year of treatment for MDD, patients in the TRD group were prescribed significantly higher doses of antidepressants (279.90 vs. 142.90 DDD, $p<0.0001$ ), lithium/mood stabilizers (DDD) (8.24 vs. 7.26 DDD, $p<0.0001)$, antipsychotics (26.63 vs. 23.79 DDD, 
Table 1 Lifetime demographic and clinical characteristics between non-TRD and TRD patients

\begin{tabular}{|c|c|c|c|c|c|}
\hline \multirow{2}{*}{$\begin{array}{l}(N=31,422) \\
\text { Variable }\end{array}$} & \multicolumn{2}{|c|}{$\begin{array}{l}\text { non-TRD group } \\
(N=20,344) 64.74 \%\end{array}$} & \multicolumn{2}{|c|}{$\begin{array}{l}\text { TRD group } \\
(N=11,078) 35.26 \%\end{array}$} & \multirow[t]{2}{*}{$P$ value ${ }^{*}$} \\
\hline & $\mathrm{N}$ & $\%$ & $\mathrm{~N}$ & $\%$ & \\
\hline Female & 8048 & 39.56 & 6673 & 60.24 & $<0.0001$ \\
\hline Urbanization level residence $^{a}$ & & & & & 0.0014 \\
\hline$|-|||$ & 16,188 & 79.61 & 8985 & 81.12 & \\
\hline$|\mathrm{V}-\mathrm{V}| \mathrm{I}$ & 4147 & 20.4 & 2092 & 18.88 & \\
\hline Estimated income via insurance (no income) & 3262 & 16.3 & 2391 & 21.82 & $<0.0001$ \\
\hline All-cause mortality & 2653 & 13.04 & 1464 & 13.22 & 0.6611 \\
\hline \multicolumn{6}{|l|}{ Lifetime physical comorbidity } \\
\hline Diabetes mellitus & 3183 & 15.65 & 2690 & 24.28 & $<0.0001$ \\
\hline Systemic lupus erythematosus & 95 & 0.47 & 113 & 1.02 & $<0.0001$ \\
\hline Rheumatoid arthritis & 308 & 1.51 & 356 & 3.21 & $<0.0001$ \\
\hline Cardiovascular disease & 3607 & 17.73 & 2805 & 25.32 & $<0.0001$ \\
\hline Renal disease & 1829 & 8.99 & 1330 & 12.01 & $<0.0001$ \\
\hline Functional gastrointestinal disorders & 5250 & 25.81 & 4660 & 42.07 & $<0.0001$ \\
\hline Thyroid dysfunction & 922 & 4.53 & 1058 & 9.55 & $<0.0001$ \\
\hline \multicolumn{6}{|l|}{ Lifetime psychiatric comorbidity } \\
\hline Personality disorders & 3065 & 15.07 & 2696 & 24.34 & $<0.0001$ \\
\hline Attention deficit hyperactivity disorder & 103 & 0.51 & 57 & 0.51 & 0.9219 \\
\hline Substance use disorders & 3385 & 16.64 & 3659 & 33.03 & $<0.0001$ \\
\hline Anxiety disorders & 13,163 & 64.7 & 9952 & 89.84 & $<0.0001$ \\
\hline Panic disorder & 1224 & 6.02 & 1732 & 15.63 & $<0.0001$ \\
\hline Non-organic psychosis & 6646 & 32.67 & 5320 & 48.02 & $<0.0001$ \\
\hline Severe depression* & 9338 & 45.90 & 6836 & 61.71 & $<0.0001$ \\
\hline Converted diagnosis to bipolar disorder & 2150 & 10.57 & 2496 & 22.53 & $<0.0001$ \\
\hline \multirow[t]{2}{*}{ Converted diagnosis to schizophrenia } & 1502 & 7.38 & 1053 & 9.51 & $<0.0001$ \\
\hline & Mean & SD & Mean & SD & \\
\hline Age of onset for depression & 37.85 & 18.82 & 41.31 & 15.33 & $<0.0001$ \\
\hline Age of onset for TRD & $\ldots$ & $\ldots$ & 44.62 & 15.60 & \\
\hline Numbers of lifetime admissions for MDD & 1.43 & 1.79 & 3.50 & 4.69 & $<0.0001$ \\
\hline Numbers of lifetime outpatient visits for MDD & 18.68 & 31.28 & 73.29 & 71.56 & $<0.0001$ \\
\hline Overall medical care utilization for all subspecialties ${ }^{b}$ & 2.40 & 4.09 & 6.85 & 15.17 & $<0.0001$ \\
\hline Days from depression to bipolar disorder & 1154.95 & 1080.22 & 1704.64 & 1125.91 & $<0.0001$ \\
\hline Days from depression to TRD & $\ldots$ & $\ldots$ & 1211.53 & 1004.66 & \\
\hline
\end{tabular}

TRD treatment-resistant depression, MDD major depression

Note ${ }^{\text {a }}$ Seven levels, with one being the most urbanized and seven being the least urbanized

Note ${ }^{b}$ Overall medical care utilization represented numbers of admissions and emergency room visits

Note ${ }^{*} P$ value thresthold $<0.002$ for Bonferroni correction

$p<0.0001)$, as well as benzodiazepines and $\mathrm{Z}$ Drugs (528.70 vs. $260.41 \mathrm{DDD}, p<0.0001$ ) than patients in the non-TRD group. Within 1 year of MDD onset, patients with TRD were also treated more frequently with combined regimens (antidepressants with either antipsychotics or anticonvulsants) than non-TRD patients $(16.44 \%$ vs. $10.14 \%, p<0.0001)$. Patients in the TRD group tended to have more outpatients visits than patients in the non-TRD group 1 year before or after the onset of MDD (psychiatric clinics, mean: 18.87 vs. $11.95, p<0.0001$; non-psychiatric clinics, mean: 50.09 vs. $42.31, p<0.0001$ ).

The results of the multiple Cox regression model are shown in Table 3. The model was adjusted for demographic features, including the age of onset, sex, geographic area, urbanization level, and income estimated by insurance. Significant variables were female gender, severe depression, lifetime psychiatric comorbidities (anxiety disorders, SUDs, personality disorders, and non- 
Table 2 Early comorbidities, prescription pattern, and behavior of seeking medical care between non-TRD and TRD patients

\begin{tabular}{|c|c|c|c|c|c|}
\hline \multirow{2}{*}{$\begin{array}{l}(N=31,422) \\
\text { Variable }\end{array}$} & \multicolumn{2}{|c|}{$\begin{array}{l}\text { non-TRD group } \\
(N=20,344) 64.74 \%\end{array}$} & \multicolumn{2}{|c|}{$\begin{array}{l}\text { TRD group } \\
(N=11,078) 35.26 \%\end{array}$} & \multirow[t]{2}{*}{$P$ value $^{*}$} \\
\hline & $\mathrm{N}$ & $\%$ & $\mathrm{~N}$ & $\%$ & \\
\hline \multicolumn{6}{|l|}{ Early physical comorbidity ${ }^{a}$} \\
\hline Diabetes mellitus & 2182 & 10.73 & 1459 & 13.17 & $<0.0001$ \\
\hline Systemic lupus erythematosus & 60 & 0.29 & 63 & 0.57 & $<0.0001$ \\
\hline Rheumatoid arthritis & 178 & 0.87 & 135 & 1.22 & 0.0034 \\
\hline Cardiac vascular disease & 2482 & 13.97 & 1943 & 17.54 & $<0.0001$ \\
\hline Renal disease & 2689 & 13.22 & 1773 & 16 & 0.0018 \\
\hline Functional gastrointestinal disorders & 3639 & 17.89 & 2682 & 24.21 & $<0.0001$ \\
\hline Thyroid dysfunction & 556 & 2.73 & 515 & 4.65 & $<0.0001$ \\
\hline \multicolumn{6}{|l|}{ Early psychiatric comorbidity ${ }^{a}$} \\
\hline Personality disorders & 2164 & 10.64 & 924 & 8.34 & $<0.0001$ \\
\hline Attention deficit hyperactivity disorder & 29 & 0.14 & 11 & 0.10 & 0.3043 \\
\hline Substance using disorders & 2153 & 10.58 & 1737 & 15.68 & $<0.0001$ \\
\hline Anxiety disorders & 11,994 & 58.96 & 9026 & 81.48 & $<0.0001$ \\
\hline Panic disorder & 727 & 3.57 & 721 & 6.51 & $<0.0001$ \\
\hline Non-organic psychosis & 2438 & 11.98 & 801 & 7.23 & $<0.0001$ \\
\hline Converted diagnosis to $\mathrm{BpD}$ within 1 year of onset & 2087 & 10.26 & 1457 & 13.15 & $<0.0001$ \\
\hline Severe depression within 1 year of onset & 6910 & 33.97 & 2637 & 23.8 & $<0.0001$ \\
\hline \multicolumn{6}{|l|}{ Prescription pattern within 1 year of onset } \\
\hline \multirow[t]{2}{*}{ Antidepressants plus antipsychotics or anticonvulsants } & 2062 & 10.14 & 1821 & 16.44 & $<0.0001$ \\
\hline & Mean & SD & Mean & SD & \\
\hline Dosage of antidepressants (DDD) & 142.90 & 152.90 & 279.90 & 223.40 & $<0.0001$ \\
\hline Dosage of lithium/mood stabilizers (DDD) & 7.26 & 34.02 & 8.24 & 34.14 & $<0.0001$ \\
\hline Dosage of antipsychotics (DDD) & 23.79 & 61.72 & 26.63 & 64.09 & $<0.0001$ \\
\hline Dosage of benzodiazepines and Z drugs (DDD) & 260.41 & 324.74 & 528.70 & 529.58 & $<0.0001$ \\
\hline \multicolumn{6}{|l|}{ Health-seeking behavior for psychiatric medical care ${ }^{b}$} \\
\hline Numbers of outpatient visits & 11.95 & 9.75 & 18.87 & 13.78 & $<0.0001$ \\
\hline \multicolumn{6}{|l|}{ Health seeking behavior for non-psychiatric medical care ${ }^{b}$} \\
\hline Numbers of outpatient visits & 42.31 & 41.36 & 50.09 & 50.07 & $<0.0001$ \\
\hline Numbers of different physicians that patients visited & 15.80 & 10.68 & 19.15 & 11.73 & $<0.0001$ \\
\hline
\end{tabular}

$T R D$ treatment-resistant depression, $B p D$ bipolar disorder, $D D D$ defined daily dose

Note ${ }^{a}$. Patients had comorbidity before depression onset or within 1 year after onset of depression

Note $^{\mathrm{b}}$. Duration was within 1 year prior or after onset of depression

Note ${ }^{*}$. $P$ value threshold $<0.002$ for Bonferroni correction

organic psychosis), lifetime physical comorbidities (diabetes, FGIDs, and thyroid dysfunction), conversion of the diagnosis to $\mathrm{BpD}$, the daily dosage of antidepressants within 1 year of onset, and non-psychiatric outpatient visits 1 year before or after the onset of depression. Anxiety disorders exhibited the strongest effect associated with TRD ( $\mathrm{aHR}=2.02$, 95\% CI: 1.89-2.16), followed by the conversion of the diagnosis to $\mathrm{BpD}(\mathrm{aHR}=1.68,95 \%$ CI: $1.61-1.75$, SUDs (aHR $=1.38,95 \%$ CI: $1.33-1.44)$, female sex (aHR $=1.24,95 \% \mathrm{CI}$ : 1.19-1.29), and personality disorders $(\mathrm{aHR}=1.23,95 \% \mathrm{CI}: 1.17-1.29)$. The PAF of each comorbidity for TRD was calculated using aHR from the Cox regression model. The most common PAFs were lifetime anxiety disorders (42.87\%), severe depression (10.59\%), SUDs (7.85\%), non-organic psychosis (6.75\%), and FGIDs (5.65\%). The average daily dose of antidepressants within 1 year since the onset of MDD $(\mathrm{aHR}=1.002,95 \%$ CI: $1.002-1.002$, every $\mathrm{DDD})$, the average daily doses of benzodiazepines and $\mathrm{Z}$ drugs $(\mathrm{aHR}=1.002,95 \% \mathrm{CI}: 1.002-1.002$, every $10 \mathrm{DDD})$, and the number of non-psychiatric outpatient visits 1 year before or after the onset of depression $(\mathrm{aHR}=1.001$, 95\% CI: $1.000-1.001)$ were also significantly associated with an increased risk of TRD. 
Table 3 Multivariable Cox regression ${ }^{\mathrm{a}}$ analysis for risk of treatment-resistant depression

\begin{tabular}{|c|c|c|c|c|}
\hline \multirow{2}{*}{$\begin{array}{l}\text { Variable } \\
\text { Age of onset (depression) ** }\end{array}$} & \multirow{2}{*}{$\begin{array}{l}\text { aHR } \\
1.007\end{array}$} & \multicolumn{2}{|c|}{$95 \% \mathrm{Cl}$} & \multirow[t]{2}{*}{ PAF } \\
\hline & & 1.005 & 1.008 & \\
\hline Female** & 1.24 & 1.19 & 1.29 & \\
\hline \multicolumn{5}{|l|}{ Lifetime physical comorbidities } \\
\hline Diabetes mellitus* & 1.09 & 1.04 & 1.14 & $1.65 \%$ \\
\hline Cardiovascular disease & 0.96 & 0.91 & 1.01 & \\
\hline Renal disease & 0.96 & 0.90 & 1.02 & \\
\hline Functional gastrointestinal disorders** & 1.19 & 1.15 & 1.24 & $5.65 \%$ \\
\hline Thyroid dysfunction** & 1.14 & 1.07 & 1.22 & $0.87 \%$ \\
\hline \multicolumn{5}{|l|}{ Lifetime psychiatric comorbidities } \\
\hline Personality disorders** & 1.23 & 1.17 & 1.29 & $4.05 \%$ \\
\hline Substance use disorders** & 1.38 & 1.33 & 1.44 & $7.85 \%$ \\
\hline Anxiety disorders** & 2.02 & 1.89 & 2.16 & $42.87 \%$ \\
\hline Non-organic psychosis** & 1.19 & 1.14 & 1.24 & $6.75 \%$ \\
\hline Severe depression** & 1.23 & 1.17 & 1.29 & $10.59 \%$ \\
\hline Converting diagnosis to bipolar disorder** & 1.68 & 1.61 & 1.75 & \\
\hline \multicolumn{5}{|l|}{ Prescription pattern within 1 year of onset } \\
\hline Dosage of antidepressants (defined daily dose)* * & 1.002 & 1.002 & 1.002 & \\
\hline $\begin{array}{l}\text { Dosage of benzodiazepine and its related drugs } \\
\text { (every } 10 \text { defined daily dose)*** }\end{array}$ & 1.002 & 1.002 & 1.002 & \\
\hline Numbers of non-psychiatric outpatient visits within 1 year prior or after onset of depression* & 1.001 & 1.000 & 1.001 & \\
\hline
\end{tabular}

*: $p$-value $<0.001 ; * *$ : $p$-value $<0.0001$

$\mathrm{Cl}$ confidence Interval, PAF population attributable fraction, aHR adjusted hazard ratio

Note ${ }^{a}$ Model adjusted for demographic features including geographic area, urbanization level, and income estimated via insurance

The results of the survival analyses are shown in Fig. 2. A significant difference in sex was identified between the groups $(p<0.0001)$. Physical comorbidities, including FGIDs and thyroid dysfunction, were associated with a higher frequency of TRD diagnosis during follow-up $(p<0.0001)$. Surprisingly, patients with early severe depressive symptoms had better prognosis than those without early severe symptoms $(p<0.0001)$. The survival rate was significantly reduced in patients with more than one comorbid psychiatric condition. The 10-year survival rate was approximately $31 \%$ in patients with multiple psychiatric comorbidities (anxiety disorders, SUDs, and personality disorders) compared with those with no prior psychiatric comorbidities (85\%).

\section{Discussion}

Using a nationwide database in the current study, one of three MDD patients have TRD. Furthermore, our results showed that patients with multiple psychiatric comorbidities have a lower 'survival rate' (not developing TRD) than those without these conditions ( $31 \%$ vs. $85 \%)$. The large registry-based claims data are generally representative as the registration rate is high (97\%) and all data regarding prescribed medications are documented. This type of study is particularly beneficial when long-term clinical management is recorded, including the duration of depressive episodes within a pre-specified patient population and clinical characteristics (e.g., comorbid conditions) $[6,32]$. However, claims data may not include the assessment of subjective treatment response and treatment adherence, and a poor response to medication may be underestimated. The definition of TRD in the current study was adapted from a previous study of claims data and was determined based on whether the patient's antidepressant treatment regimen was altered two or more times [5-7]. However, we cannot dismiss the possibility that some patients who had continued taking medication still experienced severe depression. To complement the criteria of altering medication, this definition was further added: if the patient received two or more adequate antidepressant treatment regimens but was subsequently admitted in a psychiatric acute ward. This amended definition may represent a useful proxy to capture treatment failure and allow for better identification of TRD in patients who remain in clinical settings [7, 33, 34].

The current study identified the female sex as a risk factor of TRD $(\mathrm{aHR}=1.24)$. A cross-sectional study in the UK that used questionnaires to collect treatment history found that $70 \%$ of patients with TRD were females [34], which is consistent with our findings. Furthermore, increased vulnerability to depression in women begins at puberty and declines after menopause [35]. One possible 

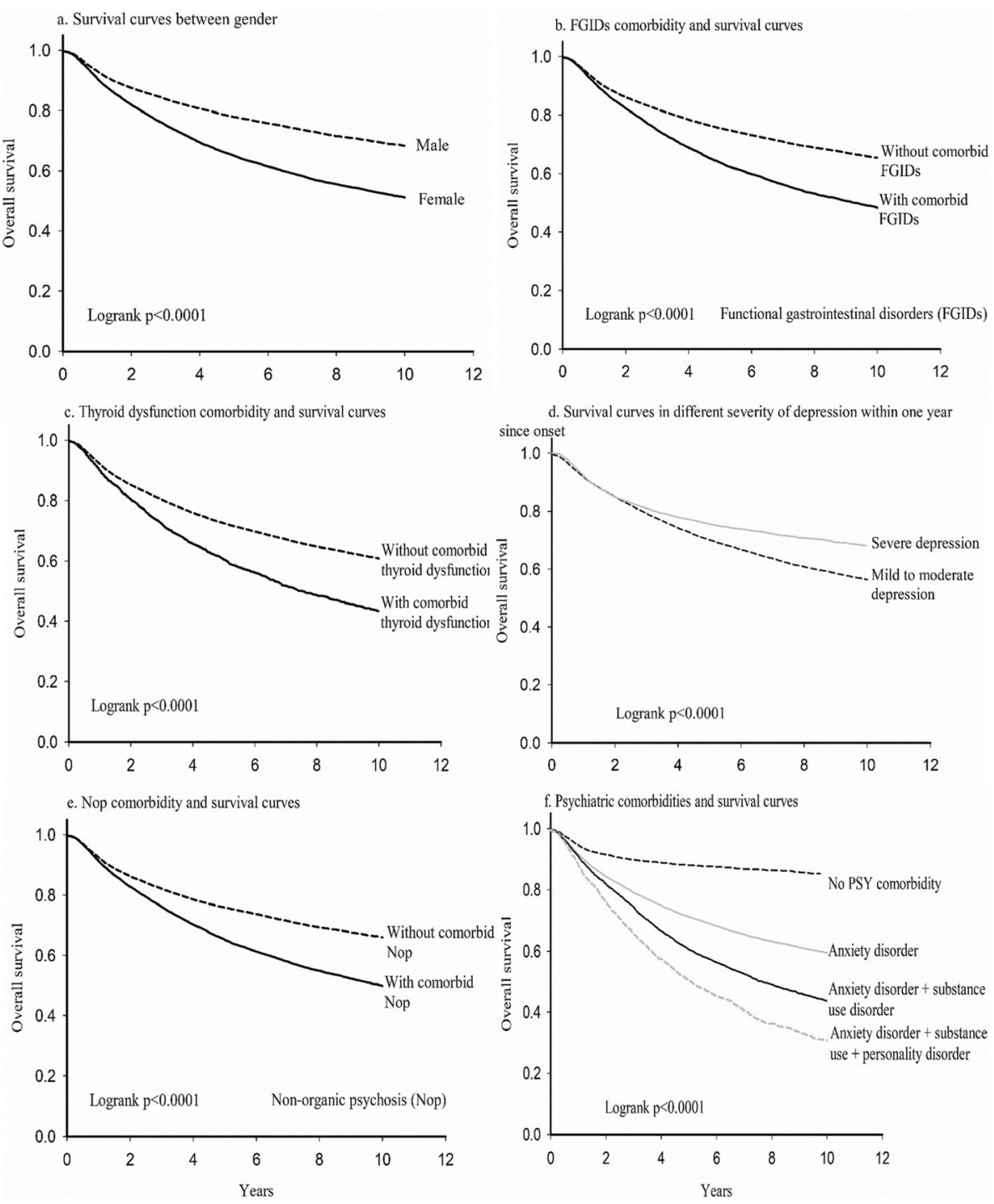

Fig. 2 Survival curves of TRD in different models. 2a) for gender; 2b) for functional gastrointestinal disorders; 2c) for thyroid dysfunction; 2d) for severe depression within 1st year since onset; 2e) for nonorganic psychosis; 2f) for different psychiatric comorbidities

explanation is that the hypothalamic-pituitary-adrenal function (cortisol levels) is more likely to fluctuate in response to stressors and during depressive episodes in women [36] . Redox imbalance elicited by estrogen has been revealed to weaken enzymatic antioxidant defenses, which may be linked to TRD $[37,38]$. Although several physical comorbidities increase the risk of depression [39], it is unknown whether these comorbidities increase the risk of TRD. The current study illustrated that patients with TRD had more physical comorbidities than those without TRD during long-term follow-up, including diabetes, FGIDs, and thyroid dysfunction. Growing evidence suggests a bidirectional communication between the gastrointestinal tract and brain [40]. An unhealthy gastrointestinal tract may result in treatment complications and reduce responsiveness to antidepressant treatment. Other studies have found that antidepressants may be used to treat FGIDs [41]. Previous studies have also indicated that thyroid dysfunction, which is more prevalent in women, also influences treatment outcomes [37, 42]. The aHR of these physical comorbidities ranged from 1.09 (diabetes mellitus) to 1.19 (FGIDs). Taken together, these risk factors have a mild yet significant impact on the development of TRD and required further study.

We reported that long-term psychiatric comorbidities are important and independent risk factors of TRD development. In particular, anxiety disorders were observed in $>80 \%$ of MDD patients in this nationwide database. We reported a 2.02-fold higher risk of TRD development in patients with comorbid anxiety disorders. In addition, our 
results revealed that anxiety is the strongest predictor of TRD with a high PAF. It is well-known that anxiety and depression/TRD often coexist [22, 23, 25], and that anxiety disorders have been identified as a risk factor of poor treatment response [43]. A previous study also found that anxiety disorders, particularly panic disorder, are associated with TRD [44]. There is evidence that depression and anxiety disorders have additive effects on increased disability and poorer recovery from medical illness [45]. Patients with comorbid depression and anxiety have more severe somatic symptoms than those MDD patients without anxiety [46], which may predispose them to sensitive to side effects from medication and dropping out of treatment prematurely [47]. In addition, comorbid anxiety in depressed patients is associated with poorer social function [45], which may correlate with poor social support. Besides, depression with comorbid symptoms of anxiety is associated with greater suicidal ideation [45]. Possibly as a result of increased illness severity, depressed patients are more likely to be prescribed benzodiazepines if they have anxiety symptoms [48]. It was also consistent with our finding of high usage of benzodiazepines use, because these drugs were prescribed two-fold more often in the TRD group than in the non-TRD group. This suggests that even in the early phase of MDD, comorbid anxiety disorders represent a red-flag in clinical settings that increases the difficulty of patient care and management and increases the risk of TRD development.

Except anxiety disorders, there are several psychiatric comorbidities correlated with TRD. A previous study found that certain personality traits, such as low cooperativeness and high neuroticism, positively correlate with TRD [13]. Personality disorders correlate with poor drug adherence, poor social function, and more SUDs, which may further result in TRD [49]. Our findings are in line with the observation that personality disorders are associated with a 1.23-fold higher risk of TRD. In addition, SUDs represent another important comorbidity condition of MDD [50]. One review article has found that mood disorders are common in patients with SUDs, whereas in many individuals, the mood disturbance cannot be attributed to the acute effects of substance use or withdrawal [51]. Mood disorders may trigger individuals to use drugs and alcohol to cope with negative affective states. Substance use is often viewed as a self-medication method to alleviate depression or anxiety. Meanwhile, substance use may mask underlying mood disturbances and aggravate symptoms, ultimately leading to TRD [52]. SUDs are also risk factors of several psychiatric disorders, including anxiety disorders; therefore, the effect on the increased risk of TRD may be confounded by SUDs. After adjustment in multiple Cox regression models, we reported that several psychiatric disorders remained independent risk factors.
The relationships between comorbidities and TRD have been examined in a cross-sectional manner without clear temporal consequences in the literature $[6,12,13]$. In the present study, using Cox regression models and plots with survival function, our results demonstrated the temporal relationship that early and long-term psychiatric comorbidities significantly increase the risk of subsequent treatment resistance events. The aHRs of psychiatric comorbidities ranged from 1.19 to 2.02; the point estimates are generally higher than those of physical comorbidities. Furthermore, approximately $70 \%$ of patients with multiple lifetime psychiatric comorbidities developed TRD in this study. On the other hand, non-organic psychosis, and severe depression at the first year of diagnosis reduced the risk of TRD. It is possible that patients with initial severe and psychotic depression may have been treated more thoroughly or prescribed with a higher dose of antidepressant medication. Taken together, the comorbidity profiles of TRD are important and warrant further evaluation for the early detection of TRD.

In the current study, the patients in the TRD group visited non-psychiatric clinics more often than those in the non-TRD group. Patients in the TRD group more commonly visited non-psychiatric clinics than psychiatric clinics during their first year of treatment. Interestingly, $65 \%$ of patients with depression sought help at general medical clinics prior to a psychiatric clinic [53]. Another clinical study reported that somatic symptoms prevailed in a great majority of depressed patients; these patients may initially seek non-psychiatric medical care before psychiatric care [54]. Patients with depression may delay proper psychiatric treatment, resulting in an increased risk of treatment resistance. Therefore, it is important to evaluate patients with depression who have frequently visit nonpsychiatric clinics and provide them suitable treatment. Furthermore, both health-seeking behavior and drug compliance may be correlated with treatment resistance. Adherence was found to play an important role in TRD development and increased the risk of mortality [8, 14, 26]. In the claims database, adherence was not easy to assess. On the other hand, patients with very poor drug adherence were unlikely to fulfill the definition of TRD for having a number of antidepressant trials. Our analysis revealed no significant difference in the mortality rate between the TRD and non-TRD groups, possibly due to the relatively young age of the patients and relatively short follow-up period in the database.

We also found that patients in the TRD group had significantly higher doses of psychotropic prescriptions and a greater incidence of long-term use than those in the nonTRD group within 1 year of MDD diagnosis. The antidepressants dose remained significantly associated with TRD in multiple analysis. Long-term treatment with antidepressants might have contributed to the subsequent treatment 
resistance [55]. There are some possible explanations for this phenomenon. First, treating patients with mood disorders using antidepressants may have a paradoxical effect (exacerbate depression) [56, 57]. Second, previous studies have described antidepressant-induced switching and cycle acceleration in patients with $\mathrm{BpD}$ [58]. In the current study, patients whose diagnosis was converted to $\mathrm{BpD}$ experienced a 1.72-fold higher risk of developing TRD. Third, tolerance to antidepressants has been reported [59]. Patients with TRD also had more complex treatment regimens, because it is a common strategy to use higher doses and adjunctive psychotropics to counter poor response to medication [60]. A recent meta-analysis of placebo-controlled trials demonstrated that adjunctive antipsychotics were effective for the treatment of TRD [61]. Another metaanalysis demonstrated the efficacy of lithium augmentation with antidepressants compared with the placebo for the treatment of TRD [62]. However, because the current study was not a randomized, placebo-controlled trial, we did not compare a combined treatment regimen with monotherapy. Rather, we found that patients eventually developing TRD required early attention and more complex treatment at an early disease stage. The results of the current study suggest that early prescription patterns in patients represents a proxy for the combined treatment regimen and poor response/prognosis.

Current treatment regimens for depression sometimes have limited efficacy. Novel antidepressants with different mechanisms are currently used as treatment options for TRD, including agents targeting the hypothalamic-pituitary-adrenal axis, glutamate/GABA system, or opioid receptors [63]. One promising agent is ketamine, which is a noncompetitive NMDA receptor antagonist that can produce rapid and stable antidepressant effects [64]. Several ongoing clinical trials of ketamine for the treatment of TRD are ongoing in Taiwan; they have revealed satisfactory effects $[65,66]$. Other widely discussed antidepressant agent are cannabinoid compounds because the endocannabinoid system is widely distributed throughout the body and serves to modulate mood symptoms [67].

This study has several limitations that need to be considered when interpreting the results. First, structured interview data were not available because we solely relied on claims data. Furthermore, more detailed clinical features and the assessments of disease severity were not available. Second, we could not obtain information on uninsured subjects. However, this study is highly representative of the target population because approximately $97 \%$ of the population was insured. Third, we targeted patients who had ever been admitted to a hospital because of depressive episodes. The results may not be extrapolated to all MDD patients, particularly to those who had never been admitted. Fourth, it was not possible to evaluate patients' true treatment compliance.
Nevertheless, we attempted to exclude those with poor compliance (e.g., patients filling prescriptions for less than 56 days). Fifth, we intended to investigate the early features of the TRD group; however, we did not have information on the subjects before they sought medical help. Sixth, we did not consider the patient's lifestyle and personal history (i.e., coping strategies, exercise habits, smoking, psychological trauma, etc.), which might have influenced the incidence of TRD. Finally, the mortality rate may be underestimated because death records were missing from the dataset and we cannot clarify the primary causes of death. Therefore, future studies are warranted to link this database with national death records to answer such questions.

\section{Conclusions}

At least one of three ever admitted patients with MDD developed TRD in this study. These patients visited nonpsychiatric medical care more frequently than specialized psychiatric facilities and were prescribed higher doses of psychotropic agents within the first year of MDD diagnosis. In addition, patients with TRD had lower incomes and utilized medical services more frequently than those without TRD. Female patients exhibited a higher risk of TRD than male patients. Our findings indicated that physical and psychiatric comorbidities substantially increase the risk of TRD during follow-up, and that $70 \%$ of patients with multiple psychiatric comorbidities developed TRD during follow-up. Clinicians should be more aware of patients' physical and psychiatric comorbidity patterns and early polypharmacy to better develop comprehensive treatment plans and minimize the risk of developing TRD.

\section{Abbreviations}

TRD: Treatment-resistant depression; MDD: Major depressive disorder; ER: Emergency room; FGIDs: Functional gastrointestinal disorders; SLE: Systemic lupus erythematosus; ADHD: Attention deficit hyperactivity disorder; SUDs: Substance use disorders; PAF: Population attributable fraction; PIMC: Psychiatric Inpatients Medical Claim; BpD: Bipolar disorder; DDD: Defined daily doses; aHRs: Adjusted hazard ratios; Cl: Confidence interval

\section{Acknowledgements \\ The statistical analysis is partially supported by the Biostatistics Task Force of National Taiwan University.}

\section{Authors' contributions \\ Study concept and design: SSH, PHK, HHC, WJC; Acquisition of data: SSH, JW; Analysis and interpretation of data: SSH, PHK, HCC; Drafting of the manuscript: $\mathrm{SSH}, \mathrm{PHK}, \mathrm{HHC}$; Critical revision of the manuscript: SSH, PHK, HHC. The author(s) read and approved the final manuscript.}

\section{Funding}

This study was supported by a grant from the Ministry of Science and Technology (NSC101-3114-Y-002-003) to Prof. Ming-Je Tang, and a grant MOST 108-2314-B-002-136-MY3 to Dr. Po-Hsiu Kuo. The funding agent had no role in study design, data collection and analysis, decision to publish, or preparation of the manuscript. 


\section{Availability of data and materials}

The datasets generated and/or analyzed of the current study are not publicly available due to the privacy policy of statistics department of Ministry of Health and Welfare, Taiwan.

\section{Ethics approval and consent to participate}

The study received ethical approval from the Institutional Review Board (IRB) of National Taiwan University (NTUH-REC-201303020RINC).

\section{Consent for publication}

Not applicable. This study uses unidentifiable data.

\section{Competing interests}

The authors declare that they have no competing interests.

\section{Author details}

${ }^{1}$ Department of Public Health \& Institute of Epidemiology and Preventive Medicine, National Taiwan University, Taipei, Taiwan. ${ }^{2}$ Bali Psychiatric Center, Ministry of Health and Welfare, New Taipei City, Taiwan. ${ }^{3}$ Department of Psychiatry, College of Medicine, National Yang-Ming University, Taipei, Taiwan. ${ }^{4}$ Taipei Veterans General Hospital, Taipei, Taiwan. ${ }^{5}$ Department of Psychiatry, Yang Ji Mental Hospital, Keelung, Taiwan. ${ }^{6}$ Department of Psychiatry, National Taiwan University Hospital, Taipei, Taiwan. ${ }^{7}$ National Health Research Institutes, Center for Neuropsychiatric Research, Miaoli County, Taiwan.

Received: 28 May 2020 Accepted: 27 October 2020

Published online: 17 November 2020

\section{References}

1. Bromet E, Andrade LH, Hwang I, Sampson NA, Alonso J, de Girolamo G, de Graaf R, Demyttenaere K, Hu C, Iwata N, et al. Cross-national epidemiology of DSM-IV major depressive episode. BMC Med. 2011;9:90.

2. Bschor T. Therapy-resistant depression. Expert Rev Neurother. 2010;10(1):7786.

3. Vos T, Barber R, Bell B, Bertozzi-Villa A, Biryukov S, Bolliger I, Charlson F. Global, regional, and national incidence, prevalence, and years lived with disability for 301 acute and chronic diseases and injuries in 188 countries, 1990-2013: a systematic analysis for the global burden of disease study 2013. Lancet. 2015:386(9995):743-800.

4. Agency (EMEA) EM. Concept Paper on the Need for Revision of Note for Guidance on Clinical Investigation of Medicinal Products in the Treatment of Depression with regard to Treatment Resistant Depression. In: Committee for Medicinal Product for Human Use (CHMP); 2009.

5. Rush AJ, Trivedi MH, Wisniewski SR, Nierenberg AA, Stewart JW, Warden D, Niederehe G, Thase ME, Lavori PW, Lebowitz BD, et al. Acute and longerterm outcomes in depressed outpatients requiring one or several treatment steps: a STAR*D report. AJ Psychiatry. 2006;163(11):1905-17.

6. Souery D, Oswald P, Massat I, Bailer U, Bollen J, Demyttenaere K, Kasper S, Lecrubier Y, Montgomery S, Serretti A, et al. Clinical factors associated with treatment resistance in major depressive disorder: results from a European multicenter study. J Clin Psychiatry. 2007;68(7):1062-70.

7. Li CT, Bai YM, Huang YL, Chen YS, Chen TJ, Cheng JY, Su TP. Association between antidepressant resistance in unipolar depression and subsequent bipolar disorder: cohort study. Br J Psychiatry. 2012;200(1):45-51.

8. Nemeroff CB. Prevalence and management of treatment-resistant depression. J Clin Psychiatry. 2007;68(Suppl 8):17-25.

9. Kautzky A, Dold M, Bartova L, Spies M, Kranz GS, Souery D, Montgomery S, Mendlewicz J, Zohar J, Fabbri C, et al. Clinical factors predicting treatment resistant depression: affirmative results from the European multicenter study. Acta Psychiatr Scand. 2019;139(1):78-88.

10. Peele $\mathrm{PB}, \mathrm{X} \cup \mathrm{Y}, \mathrm{Kupfer} \mathrm{DJ}$. Insurance expenditures on bipolar disorder: clinical and parity implications. AJ Psychiatry. 2003;160(7):1286-90.

11. Kohler S, Wiethoff K, Ricken R, Stamm T, Baghai TC, Fisher R, Seemuller F, Brieger $\mathrm{P}$, Cordes J, Malevani J, et al. Characteristics and differences in treatment outcome of inpatients with chronic vs. episodic major depressive disorders. J Affect Disord. 2015;173:126-33.

12. Dudek D, Rybakowski JK, Siwek M, Pawlowski T, Lojko D, Roczen R, Kiejna A. Risk factors of treatment resistance in major depression: association with bipolarity. J Affect Disord. 2010;126(1-2):268-71.
13. Takahashi M, Shirayama Y, Muneoka K, Suzuki M, Sato K, Hashimoto K. Low openness on the revised NEO personality inventory as a risk factor for treatment-resistant depression. PLoS One. 2013;8(9):e71964.

14. Krivoy A, Balicer RD, Feldman B, Hoshen M, Zalsman G, Weizman A, Shoval $\mathrm{G}$. Adherence to antidepressants is associated with lower mortality: a 4-year population-based cohort study. J Clin Psychiatry. 2016;77(5):e566-72.

15. Simayi A, Mohemaiti P. Risk and protective factors of co-morbid depression in patients with type 2 diabetes mellitus: a meta analysis. Endocr J. 2019; 66(9):793-805

16. Chireh B, Li M, D'Arcy C. Diabetes increases the risk of depression: a systematic review, meta-analysis and estimates of population attributable fractions based on prospective studies. Prev Med Rep. 2019;14:100822.

17. Doering LV, Chen B, McGuire A, Bodan RC, Irwin MR. Persistent depressive symptoms and pain after cardiac surgery. Psychosom Med. 2014;76(6):43744.

18. Lewis-Fernandez R, Lam P, Lucak S, Galfalvy H, Jackson E, Fried J, Rosario M, de la Cruz AA, Sanchez-Lacay A, Diaz S, et al. An open-label pilot study of duloxetine in patients with irritable bowel syndrome and comorbid major depressive disorder. J Clin Psychopharmacol. 2016;36(6):710-5.

19. Hage MP, Azar ST. The link between thyroid function and depression. J Thyroid Res. 2012;2012:1-8.

20. MM LP, Tani C, Gemignani A, Mauri M, Bombardieri S. Depression and systemic lupus erythematosus: a systematic review. Lupus. 2013;22(5):409_ 16

21. Nerurkar L, Siebert S, Mclnnes IB, Cavanagh J. Rheumatoid arthritis and depression: an inflammatory perspective. Lancet Psychiatry. 2019;6(2):16473.

22. Rush AJ, Zimmerman M, Wisniewski SR, Fava M, Hollon SD, Warden D, Biggs MM, Shores-Wilson K, Shelton RC, Luther JF, et al. Comorbid psychiatric disorders in depressed outpatients: demographic and clinical features. J Affect Disord. 2005;87(1):43-55.

23. Kessler RC, Berglund P, Demler O, Jin R, Merikangas KR, Walters EE. Lifetime prevalence and age-of-onset distributions of DSM-IV disorders in the National Comorbidity Survey Replication. Arch Gen Psychiatry. 2005;62(6): 593-602.

24. Asp $M$, Lindqvist $D$, Fernstrom J, Ambrus $L$, Tuninger $E$, Reis $M$, Westrin $A$. Recognition of personality disorder and anxiety disorder comorbidity in patients treated for depression in secondary psychiatric care. PLoS One. 2020;15(1):e0227364

25. Cepeda MS, Reps J, Ryan P. Finding factors that predict treatment-resistant depression: results of a cohort study. Depress Anxiety. 2018;35(7):668-73.

26. Kollhorst B, Jobski K, Krappweis J, Schink T, Garbe E, Schmedt N. Antidepressants and the risk of death in older patients with depression: a population-based cohort study. PLoS One. 2019;14(4):e0215289.

27. Wu CS, Desarkar P, Palucka A, Lunsky Y, Liu SK. Acute inpatient treatment, hospitalization course and direct costs in bipolar patients with intellectual disability. Res Dev Disabil. 2013;34(11):4062-72.

28. Oquendo MA, Baca-Garcia E, Kartachov A, Khait V, Campbell CE, Richards M, Sackeim HA, Prudic J, Mann JJ. A computer algorithm for calculating the adequacy of antidepressant treatment in unipolar and bipolar depression. J Clin Psychiatry. 2003;64(7):825-33.

29. Chiang $C L$, Chen PC, Huang LY, Kuo PH, Tung YC, Liu CC, Chen WJ. Impact of universal health coverage on urban-rural inequity in psychiatric service utilisation for patients with first admission for psychosis: a 10-year nationwide population-based study in Taiwan. BMJ Open. 2016;6(3): e010802.

30. McIntyre RS, Filteau MJ, Martin L, Patry S, Carvalho A, Cha DS, Barakat M, Miguelez M. Treatment-resistant depression: definitions, review of the evidence, and algorithmic approach. J Affect Disord. 2014;156:1-7.

31. Levin ML. The occurrence of lung cancer in man. Acta Unio Int Contra Cancrum. 1953:9(3):531-41.

32. Fekadu A, Wooderson S, Donaldson C, Markopoulou K, Masterson B, Poon L, Cleare AJ. A multidimensional tool to quantify treatment resistance in depression: the Maudsley staging method. J Clin Psychiatry. 2009;70(2):177-84.

33. Kubitz N, Mehra M, Potluri RC, Garg N, Cossrow N. Characterization of treatment resistant depression episodes in a cohort of patients from a US commercial claims database. PLoS One. 2013;8(10):e76882.

34. Thomas L, Kessler D, Campbell J, Morrison J, Peters TJ, Williams C, Lewis G, Wiles N. Prevalence of treatment-resistant depression in primary care: crosssectional data. Br J Gen Pract. 2013;63(617):e852-8. 
35. Van Zundert J, Hans G, van Kuijk S, Van Boxem K, Vissers K. Low back pain. Lancet. 2018;392(10164):2548-9.

36. Clark S, Horton R. Low back pain: a major global challenge. Lancet. 2018 391(10137):2302

37. Fortunato RS, Ferreira AC, Hecht F, Dupuy C, Carvalho DP. Sexual dimorphism and thyroid dysfunction: a matter of oxidative stress? Endocrinol. 2014;221(2):R31-40.

38. Sowa-Kucma M, Styczen K, Siwek M, Misztak P, Nowak RJ, Dudek D, Rybakowski JK, Nowak G, Maes M. Lipid peroxidation and immune biomarkers are associated with major depression and its phenotypes, including treatmentresistant depression and melancholia. Neurotox Res. 2018;33(2):448-60.

39. Maes M, Kubera M, Obuchowiczwa E, Goehler L, Brzeszcz J. Depression's multiple comorbidities explained by (neuro) inflammatory and oxidative \& nitrosative stress pathways. Neuro endocrinology letters. 2011;32(1):7-24.

40. Tache $Y$, Bernstein CN. Evidence for the role of the brain-gut axis in inflammatory bowel disease: depression as cause and effect? Gastroenterology. 2009:136(7):2058-61.

41. Ruepert L, Quartero AO, de Wit NJ, van der Heijden GJ, Rubin G, Muris JW. Bulking agents, antispasmodics and antidepressants for the treatment of irritable bowel syndrome. Cochrane Database Syst Rev. 2011;8:CD003460.

42. Ahlawat SK, Cuddihy MT, Locke GR 3rd. Gender-related differences in dyspepsia: a qualitative systematic review. Gend Med. 2006;3(1):31-42.

43. van Loo HM, Cai T, Gruber MJ, Li J, de Jonge P, Petukhova M, Rose S, Sampson NA, Schoevers RA, Wardenaar KJ, et al. Major depressive disorder subtypes to predict long-term course. Depress Anxiety. 2014;31(9):765-77.

44. Bennabi D, Aouizerate B, El-Hage W, Doumy O, Moliere F, Courtet P, Nieto I, Bellivier F, Bubrovsky M, Vaiva G, et al. Risk factors for treatment resistance in unipolar depression: a systematic review. J Affect Disord. 2015;171:137-41.

45. Lenze EJ, Mulsant BH, Shear MK, Alexopoulos GS, Frank E, Reynolds CF 3rd. Comorbidity of depression and anxiety disorders in later life. Depress Anxiety. 2001;14(2):86-93.

46. Lenze EJ. Comorbidity of depression and anxiety in the elderly. Curr Psychiatry Rep. 2003;5(1):62-7.

47. Flint AJ, Rifat SL. Anxious depression in elderly patients. Response to antidepressant treatment. Am J Geriatr Psychiatry. 1997;5(2):107-15.

48. Pfeiffer PN, Ganoczy D, Zivin K, Valenstein M. Benzodiazepines and adequacy of initial antidepressant treatment for depression. J Clin Psychopharmacol. 2011;31(3):360-4

49. Trull TJ, Sher KJ, Minks-Brown C, Durbin J, Burr R. Borderline personality disorder and substance use disorders: a review and integration. Clin Psychol Rev. 2000;20(2):235-53.

50. Grant BF, Stinson FS, Dawson DA, Chou SP, Dufour MC, Compton W, Pickering RP, Kaplan K. Prevalence and co-occurrence of substance use disorders and independent mood and anxiety disorders: results from the National Epidemiologic Survey on alcohol and related conditions. Arch Gen Psychiatry. 2004;61(8):807-16

51. Tolliver BK, Anton RF. Assessment and treatment of mood disorders in the context of substance abuse. Dialogues Clin Neurosci. 2015;17(2):181-90.

52. Quello SB, Brady KT, Sonne SC. Mood disorders and substance use disorder: a complex comorbidity. Sci Pract Perspect. 2005;3(1):13-21.

53. Boerema AM, Kleiboer A, Beekman AT, van Zoonen K, Dijkshoorn H, Cuijpers $P$. Determinants of help-seeking behavior in depression: a cross-sectional study. BMC Psychiatry. 2016;16:78

54. Hamilton M. Frequency of symptoms in melancholia (depressive illness). Br J Psychiatry. 1989:154:201-6.

55. Fava GA. Can long-term treatment with antidepressant drugs worsen the course of depression? J Clin Psychiatry. 2003;64(2):123-33.

56. van Scheyen JD. Recurrent vital depressions. A follow-up study of 56 female and 28 male patients. Psychiatr Neurol Neurochir. 1973;76(2):93-112.

57. Mann JJ, Kapur S. The emergence of suicidal ideation and behavior during antidepressant pharmacotherapy. Arch Gen Psychiatry. 1991;48(11):1027-33.

58. Baldessarini RJ, Faedda GL, Offidani E, Vazquez GH, Marangoni C, Serra G, Tondo L. Antidepressant-associated mood-switching and transition from unipolar major depression to bipolar disorder: a review. J Affect Disord. 2013;148(1):129-35.

59. Katz G. Tachyphylaxis/tolerance to antidepressants in treatment of dysthymia: results of a retrospective naturalistic chart review study. Psychiatry Clin Neurosci. 2011;65(5):499-504.

60. de Sousa RT, Zanetti MV, Brunoni AR, Machado-Vieira R. Challenging treatment-resistant major depressive disorder: a roadmap for improved therapeutics. Curr Neuropharmacol. 2015;13(5):616-35.
61. Spielmans Gl, Berman MI, Linardatos E, Rosenlicht NZ, Perry A, Tsai AC Adjunctive atypical antipsychotic treatment for major depressive disorder: a meta-analysis of depression, quality of life, and safety outcomes. PLoS Med. 2013:10(3):e1001403.

62. Nelson JC, Baumann P, Delucchi K, Joffe R, Katona C. A systematic review and meta-analysis of lithium augmentation of tricyclic and second generation antidepressants in major depression. J Affect Disord. 2014;168:269-75.

63. Wang Q, Dwivedi Y. Advances in novel molecular targets for antidepressants. Prog Neuropsychopharmacol Bol Psychiatry. 2021;104:110041.

64. Chen MH, Lin WC, Tu PC, Li CT, Bai YM, Tsai SJ, Su TP. Antidepressant and antisuicidal effects of ketamine on the functional connectivity of prefrontal cortex-related circuits in treatment-resistant depression: a double-blind, placebo-controlled, randomized, longitudinal resting fMRI study. J Affect Disord. 2019;259:15-20.

65. Su TP, Chen MH, Li CT, Lin WC, Hong CJ, Gueorguieva R, Tu PC, Bai YM, Cheng CM, Krystal JH. Dose-related effects of adjunctive ketamine in Taiwanese patients with treatment-resistant depression. Neuropsychopharmacology. 2017;42(13):2482-92.

66. Chen MH, Lin WC, Wu HJ, Bai YM, Li CT, Tsai SJ, Hong CJ, Tu PC, Cheng CM, Su TP. Efficacy of low-dose ketamine infusion in anxious vs nonanxious depression: revisiting the adjunctive ketamine study of Taiwanese patients with treatment-resistant depression. CNS Spectr. 2020:1-6.

67. Ferber SG, Namdar D, Hen-Shoval D, Eger G, Koltai H, Shoval G, Shbiro L, Weller A. The "entourage effect": Terpenes coupled with cannabinoids for the treatment of mood disorders and anxiety disorders. Curr Neuropharmacol. 2020;18(2):87-96.

\section{Publisher's Note}

Springer Nature remains neutral with regard to jurisdictional claims in published maps and institutional affiliations.

Ready to submit your research? Choose BMC and benefit from:

- fast, convenient online submission

- thorough peer review by experienced researchers in your field

- rapid publication on acceptance

- support for research data, including large and complex data types

- gold Open Access which fosters wider collaboration and increased citations

- maximum visibility for your research: over $100 \mathrm{M}$ website views per year

At $\mathrm{BMC}$, research is always in progress.

Learn more biomedcentral.com/submissions 